\title{
Cyperaceae da flora Sul-matogrossense: composição florística
}

\author{
Ana Claudia Araújo' \& Rafael Trevisan² \\ 'Natural History Museum. Life Science Department - Plants Division, Cromwell Road, SW7 5BD, London, United Kingdon. \\ anaclaudiaaraujo 1@hotmail.com \\ 2Universidade Federal de Santa Catarina, Departamento de Botânica, Trindade, CEP 88040-970, \\ Florianópolis, Santa Catarina, Brasil.
}

Recebido em 23.IX. 2014

Aceito em 22.XI.2016

DOI $10.21826 / 2446-8231201873 s 190$

RESUMO - Este estudo apresenta os resultados de investigação florística para a família Cyperaceae dentro do programa Biota do Mato Grosso do Sul, Brasil. Foi realizada uma pesquisa na literatura específica e utilizados informações de herbário, nacionais e internacionais, investigados pessoalmente e/ou eletronicamente resultando em um banco de dados, o qual é a base dos resultados aqui apresentados. Constata-se que cestá representada no estado do Mato Grosso do Sul por 21 gêneros e 183 espécies. Dos taxa listados cerca de 6 \% não foram ainda confirmadas. A família apresenta maior diversidade de espécies no Cerrado. Os gêneros com maior riqueza específica são Rhynchospora Vahl seguido de Cyperus L.

Palavras-chave: conservação, diversidade, florística, taxonomia

ABSTRACT - Cyperaceae of the Mato Grosso do Sul flora. This study presents the results of a florisitc investigation on Cyperaceae family, according to the Biota program in the Mato Grosso do Sul, Brazil. A survey based on specific literature and herbaria information, national and international, accessed personally and/or online produced a dataset from which the information here is provided. As a result, Cyperaceae is represented in the Mato Grosso do Sul state by 21 genera and 183 species. From all taxa listed here ca. of 6\% are not yet confirmed. The family is more diverse in the cerrado. The genera richer in species number are Rhynchospora Vahl, followed by Cyperus L.

Keywords: conservation, diversity, floristic, taxonomy

\section{INTRODUÇÃO}

Cyperaceae é a terceira família mais numerosa em espécies dentro das monocotiledôneas, e configura entre as 10 maiores no Neotrópico (Thomas 2004, Heywood et al. 2007). A mesma esta posicionada junto com Juncaceae e Thurniaceae em um clado monofilético dentro da ordem Poales (APG III 2009). Embora amplamente conhecidas como plantas invasoras (Lorenzi 2006) muitas espécies são consideradas pelo seu grande valor comercial, principalmente como plantas medicinais ou fontes de fibra para produtos de artesanato (Simpson 2008).

Tipicamente são ervas de pequeno a médio porte $(5-200$ $\mathrm{cm}$ ), podendo chegar a mais de $5 \mathrm{~m}$ de altura (Goetghebeur 1998, Thomas 2004, Araújo et al. 2009); estrutura caulinar lignificada também ocorre e algumas espécies apresentam desenvolvimento secundário (Rodrigues \& Estelita 2002). Espécies desta família são de difícil identificação em virtude da extrema redução floral a qual é composta, em geral, apenas pelos verticilos reprodutivos, protegidos por estruturas bracteóides, as glumas. Em alguns gêneros resquícios do perigônio estão presentes na forma de estruturas hipógenas (cerdas ou estruturas laminaris escamiformes). As flores, bissexuadas ou unissexuadas, organizam-se em pequenas espigas (espiguetas), as quais formam a unidade da inflorescência. As espiguetas podem estar organizadas em glomérulos, espigas ou em estruturas paniculiformes ou corimbiformes. $\mathrm{O}$ androceu na maioria das espécies é composto de três estames, enquanto o gineceu é formado por um ovário unilocular, uniovulado, composto por dois a três carpelos, consequentemente apresentando número equivalente de estigmas (Goetghebeur 1998, Thomas 2004, Araújo et al. 2009).

A família conta atualmente com mais de 100 gêneros reconhecidos e cerca de 5.000 espécies, pantropicais em sua maioria (Govaerts \& Simpson 2007, Heywood et al. 2007), contudo bem representadas nas áreas temperadas do globo, principalmente pelos maiores gêneros, Carex $\mathrm{L}$. (1.700-2.000 ssp) e Cyperus L. (700 ssp. aproximadamente) (Goverts \& Simpson 2007, Bruce et al. 2008). Ressaltase que Carex também é registrado em áreas subtropicais ou mesmo tropicais, geralmente em ambientes frios e úmidos em elevadas altitudes (Alves et al. 2009). Cyperus, o qual reflete a segunda melhor representação de ciperáceas 
em zonas temperadas, está ainda melhor distribuído nos trópicos, posição compartilhada com outros gêneros, tais como Rhynchospora Vahl e Scleria P.J. Bergius (Alves et al. 2009, Araujo et al. 2009, Afonso 2015). Embora comumente relacionados a ambientes preferencialmente úmidos e abertos, como é o caso de Eleocharis R. Br. (Goetghebeur 1998), várias gêneros são encontrados em ambientes abertos, porém secos Rhynchospora e Bulbostylis Kunth, enquanto outros ocupam ambientes florestais tais como Hypolytrum Rich., Pleurostachys Brongn. e Scleria (Core 1936, Alves 2003, Araújo et al. 2009).

O Brasil está representado por $10 \%$ da riqueza de espécies mundialmente conhecidas e aceitas, cerca de 600 distribuídas em 40 gêneros. Rhynchospora Vahl (136), Cyperus (86), Scleria (71) e Eleocharis R.Br. (65) são os gêneros melhor representados (Alves et al. 2013). Ainda de acordo com esses autores, a região sudeste apresenta a maior diversidade e riqueza de espécies, enquanto o Norte apresenta maior riqueza de gêneros. O CentroOeste está representado por 22 gêneros e 274 espécies, o menor número para o Brasil nesta família, (Figs 1,2). Estes mesmos autores litstam para o Mato Grosso do Sul 17 species em oito gêneros.

O primeiro estudo da flora ciperológica na região Centro-Oeste foi publicado por Dubs (1998) incluindo a flora dos estados do Mato Grosso e Mato Grosso do Sul. Na ocasião, 133 espécies de Cyperaceae foram listadas por Simpson (1998). Posteriormente, foi publicada a flora do Pantanal, incluindo o estado do Mato Grosso do Sul (Pott \& Pott 2000) e mais recentemente a família aparece na flora do Distrito Federal (Araújo et al. 2009).

Contudo, permanecia uma lacuna sobre flora do Mato Gross do Sul "per se", lacuna esta que este estudo vem agora preencher. Aqui é apresentada e discutida a listagem de espécies de Cyperaceae para o estado do Mato Grosso do Sul, as quais estão acompanhadas de voucher, dados ecológicos e estatísticos.

\section{Principais Grupos de Pesquisa}

Os ciperólogos brasileiros estão, em sua maioria, presentes no Sul e Sudeste do país, seguido do Nordeste que tem promovido estudos ciperológicos regionais da família. A dispersão de especialistas promove a distribuição de conhecimentos bem como trabalhos cooperativos, porem, sob este formato, grupos de pesquisa em um único local ainda não está estabelecido para esta família no Brasil. Primeiramente o Rio Grande do Sul, seguido de São Paulo e posteriormente, o Rio de Janeiro tornaram-se o centro de produção de ciperólogos. Mais recentemente Santa Catarina tem promovido a formação de novos especialistas. Particularmente universidades seguido de jardins botânicos promovem estudos florísticos e taxonômicos, resultado de dissertações de mestrado e teses de doutorado. Recife e Londrina lideram os estudos genéticos. Estudos moleculares avançam mais rapidamente no exterior; Estados Unidos, Bélgica, Inglaterra e África do Sul estão entre os paises que, colaborativamente, investigam o relacionamento entre diferente gêneros dentro de cada tribo aceita no momento. Estudos estruturais estão praticamente concentrados em São Paulo.

\section{Principais Lacunas de Conhecimento}

Persiste a grande lacuna de conhecimento florístico no nível específico dentro do Brasil, incluindo não somente a riqueza total de espécies, mas quem elas são, onde estão, tamanho e distribuição das respectivas populações, estado de conservação, bem como a riqueza relativa destas espécies em cada vegetação. Acrescenta-se também o desafio de ponderar o uso da biologia molecular enquanto ferramenta científica para criação de novas espécies e a rapidez destas publicações. Novos nomes vêm tornando a lista de sinônimos numerosa sem necessariamente refletir diversidade e ou riqueza científica. A deficiência de informações básicas de qualidade dificulta a avaliação correta do estado de conservação de espécies e, consequentemente, o estado de conservação das vegetações onde essas estão presentes. No Brasil, a riqueza e diversidade de Cyperaceae na região amazônica precisa ser priorizada.

Os estudos moleculares ainda são preliminares em vários taxa, as tribos Rhynchosporae e Sclereae são prioridades particularmente na flora brasileira. Estudos estruturais avançaram bastante no Brasil nos últimos anos, porém ainda são embrionários. Finalmente, várias espécies da família possuem valor econômico, porém tais informações são incompletas, o conhecimento sobre o tema ainda é pouco conhecido e /ou divulgado, bem como o que é conhecido subutilizado economicamente. A botânica econômica poderia impulsionar a economia regional e, se monitorada e manejada adequadamente, poderia impulsionar estratégias de conservação. Tais estratégias contribuiriam no conhecimento de diversidade e riqueza da família e do valor ecológico de Cyperaceae nas diversas vegetações onde a família é dominante ou bem representada. Visto que no Mato Grosso do Sul.

\section{MATERIAL E MÉTODOS}

O presente estudo foi desenvolvido utilizando-se informações resgatadas através de três vias distintas: banco de dados dos autores; informações disponibilizadas online as quais foram adquiridas seja pelo website dos herbarios investigados (F, K, MO, NY, P, US) ou herbários incorporados ao sistema CRIA (BHCB, CGMS, HUFU, INPA, MBM, RB, SP, UB, UFP); bem como literatura especializada citada na bibliografia (em certos casos elas não aparecem no texto, mas foram usadas como fonte para citação de voucher). Os herbarios são listados pelos seus respectivos códigos em concordância com Thiers (2012). Nome dos autores dos taxa aqui referidos seguem o estabelecido por Brummitt \& Powell (1992).

A lista de espécies aceitas segue Govaerts \& Simpson (2007) e Alves et al. (2013), em casos conflitantes manteve- 
se o estabelecido por Alves et al. (2013) visto que esta últma foi elaborada por especialistas que detem o conhecimento da ciperologia brasileira.

No quadro 1, as espécies ainda não confirmadas aparecem como "provável ocorrência", quando são comuns aos ambientes de cerrado ou pantanal e foram registradas nas áreas limítrofes ao Mato Grosso do Sul, como o estado do Mato Grosso ou Bolívia e como "possível ocorrência", quando as espécies são comuns no cerrado e amplamente distribuídas no Brasil.

\section{RESULTADOS E DISCUSSÃO}

\section{Diversidade florística e vegetação}

Foram listadas para o estado do Mato Grosso do Sul 183 taxa aceitos, das quais ca. de 6\% ainda não confirmadas, distribuídas em 19 gêneros (Quadro 1), além de Carex L. e Scirpus L. que estão registrados para o estado porém não identificados no nível específico, consequentemente não listados. Desta forma, a identificação dos espécimes destes dois últimos gêneros certamente aumentará o número de espécies aqui listado.

Estes resultados superam o que foi apresentado por Dubs (1998) para os estados do Mato Grosso e Mato Grosso do Sul e representa cerca de $60 \%$ das espécies listadas recentemente para todo o Centro-Oeste (Fig. 3), bem como para a vegetação do Cerrado como um todo referido por Alves et al. (2013), constituindo-se em uma valiosa contribuição no refinamento dos dados da Lista do Brasil, o qual acreditamos foi realizado (Alves et al. 2014). O presente resultado Acredita-se também que o presente estudo representa um passo largo no avanço do conhecimento florístico do estado e região.

Neste estudo a diversidade específica e génerica de Cyperaceae no Mato Grosso do Sul reflete aquela encontrada para o Brasil em que os gêneros com maior riqueza específica são Rhynchospora (49) Cyperus (35) e Eleocharis (25) seguidos de Scleria (24) e Bulbostylis (19), considerando-se todos os nomes registrados e com possível ou provável ocorrência (Fig. 3).

A riqueza específica destes gêneros poderia ser explicada com base na cobertura vegetacional do estado. Segundo Alves et al. (2013) o cerrado brasileiro é a vegetação com a segunda melhor representação de espécies de Cyperaceae, enquanto o Pantanal apresenta o menor número de espécies (Fig. 4). Este estudo revela que a vegetação pantaneira tem uma riqueza específica maior do que aquela apresentada previamente, embora confirma-se o cerrado mais rico em espécies em relação ao encontrado na vegetação pantaneira. Nos ambientes úmidos e brejosos predominam, primeiramente, Cyperus, seguido de Eleocharis e Rhynchospora. A riqueza de espécies de Cyperus se dá tanto pela presença de espécies nativas quanto de espécies exóticas naturalizadas, invasoras ou não, que ocorrem em áreas ruderais. Desta forma, o gênero está bem representado tanto em ambientes mais úmidos quanto nos mais secos. Igualmente, levando-se em conta que a área territorial do Mato Grosso do Sul está coberta em grande parte pelo Pantanal, não é surpresa confirmar Eleocharis como o terceiro gênero mais diverso no estado, refletindo sua preferêcia por ambientes mais úmidos. Contudo, vale lembrar que vastas áreas de cerrado nesse estado são sasonalmente bastante úmidas o que contribui para a boa representação de Eleocharis também nessa vegetação. Ainda com relação à Eleocharis, surpreende a presença de E. vivipara Link, até o momento conhecida apenas na região texana dos Estados Unidos, conforme indicado pelo segundo author. Este registro presume-se ser o primeiro para o Brasil (Alves et al. 2015).

Como mencionado acima, a maioria das espécies listadas ocupa áreas de campo cerrado, solo seco ou úmido, segundo domíinio geográfico mais rico em especies de Cyperaceae no nível nacional (Alves et al. 2013). No cerrado os gêneros mais diversos são, respectivamente, Rhynchospora e Bulbostylis, este último mais numeroso em ambientes abertos e relativamente secos de solo arenoso. Rhynchospora é predominante no neotrópico, resultado da eficiente capacidade de adaptação à diferentes ambientes, à variabilidade climática e vegetacional, consequentemente a riqueza de espécies deste genero no Mato Grosso do Sul era esperada. Rhynchospora eurycarpa A.C. Araújo \& LonghiWagner é endêmica do cerrado da região central. Cyperus também está bem representado no cerrado, sendo o terceiro gênero mais frequente, principalmente nos ambientes abertos de solo argiloso e úmido.

Por outro lado, a riqueza de Scleria, geralmente mais relacionado aos ambientes florestais, impressiona tanto para o cerrado quanto para o pantanal. Com base na divisão infragenérica, embora controversa, os cinco subgêneros aceitos refletem de certa forma preferências ambientais. Enquanto espécies dos subgen. Schizolepis e Scleria (s. s.) são mais frequentementes em florestas, o subgen. Ophryoscleria é mais diverso em banhados ou, neste estado, no Pantanal; e finalmente espécies do subgen. Hypoporum preferem ambientes abertos e estão mais concentradas nas áreas de campo cerrado. Neste estudo varias espécies do subgen. Scleria foram encontradas em regiões de cerrado, muito provavelmente oriundas de bordas de floresta de galeria. Três espécies do subgen. Hymenolytrum, em geral concentrado na vegetação amazônica, foram registrados no Mato Grosso do Sul. Um terço das Scleria Sul Matogrossense extendem a distribuição até a região sul do Brasil (Affonso et al. 2015)

Salienta-se que foram registrados nove coleções do gênero Carex e duas do gênero Scirpus, ainda indeterminadas no nível especeifico, o que compromete a profundidade na presente discussão. $\mathrm{O}$ número de material indeterminado mesmo em outros gêneros ainda é relativamente grande em diversos herbários, particularmente nessa região.

Surpreendentemente nota-se a ausência dos gêneros Hypolytrum Rich. e Pleurostachys Brongn. que são mais diversos na América do Sul, ambos com alto grau de endemismo e bem representados no Brasil por, respectivamente, 28 e 21 espécies (Alves et al. 2013). 
Quadro 1. Espécies de Cyperaceae listadas para o estado do Mato Grosso do Sul.

\begin{tabular}{|c|c|c|c|c|}
\hline Espécies & Habitat & Registros & Vegetação & Voucher \\
\hline Abildgaardia baeotryon A.St.-Hil. & & 1 & & Pereira, E. 512. (RB) \\
\hline A. ovata (Burm. f.) Kral & Campo cerrado, solo arenoso & 1 & Cerrado & Araújo, A.C. et al. 724 (ICN) \\
\hline Ascolepis brasiliensis (Kunth) Benth. ex C.B. Clarke & Brejo & 21 & Pantanal & Pott V.J. 6721 (CGMS) \\
\hline Becquerelia cymosa Brongn. & & & & provável ocorrência \\
\hline Bulbostylis amambayensis Barros & Campo arenoso & 2 & Cerrado & Pedersen, T.M. 15984 (NY) \\
\hline B. brevifolia Palla & Mata ciliar & 1 & Floresta & Rojas 13795 (CTES) \\
\hline B. capillaris (L.) C.B. Clarke & Campo seco & 7 & Cerrado & Fina, B.G. 380 (CGMS) \\
\hline B. conifera (Kunth) C.B. Clarke & Locais úmidos & 11 & Cerrado & Pott, A.2366 (CPAP) \\
\hline B. consanguinea (Kunth) C.B. Clarke & & 1 & Cerrado & Silva, R.H. 799 (CGMS) \\
\hline B. jacobinae (Steud.) Lindm. & Cerrado solo rochoso & 4 & Cerrado & $\begin{array}{l}\text { Pott, A. \& Pott, V.J. } 6772 \\
\text { (MBM) }\end{array}$ \\
\hline B. junciformis (Kunth) C.B. Clarke & Campo & 6 & Cerrado & $\begin{array}{l}\text { Hatschbach, G. et al. 58590a } \\
\text { (MBM) }\end{array}$ \\
\hline B. juncoides (Vahl) Kunth ex Herter & Campo & 1 & Cerrado & Conceição, C.A. 1961 (CAC) \\
\hline B. latifolia Kral \& M.T.Strong & & 1 & Cerrado & Nienstedt, E.F. s.n. (RB, US) \\
\hline B. loefgrenii (Boeck.) Prata \& M. G. López & Campo cerrado & 1 & Cerrado & Hatschbach, G. 33041 (MBM) \\
\hline B. major Palla & & & & provável ocorrência \\
\hline B. paradoxa (Spreng.) Lindm. & Campo cerrado queimado & 8 & Cerrado & Pott, A.12293 (CGMS) \\
\hline B. paraensis C.B. Clarke & Local alagado & 1 & & Pott, A. et al. 6562 (CPAP) \\
\hline B. scabra (J.Presl \& C.Presl) C.B. Clarke & & 3 & & Pott, A. 2500 (CPAP) \\
\hline B. sphaerocephala (Boeck.) C.B. Clarke & Campo cerrado & 5 & Cerrado & Hatschbach, G. 33036 (MBM) \\
\hline B. svensoniana Steymerm. & & 2 & & $\begin{array}{l}\text { Pott, A. \& V. J. Pott } 8035 \\
\text { (CPAP) }\end{array}$ \\
\hline B. tenuifolia (Rudge) J.F. Macbr. & & 1 & & Pott, V.J. 13969 (HMS) \\
\hline B. truncata (Nees) M. Strong & & 1 & & $\begin{array}{c}\text { Damasceno Jr., G.A. et al. } 2590 \\
\text { (COR) }\end{array}$ \\
\hline B. vestita (Kunth) C.B. Clarke & & 1 & & Pott, V.J. et al. (7474 (HMS) \\
\hline Calyptrangium verticillatum (Spreng.) Vitta & Cerrado solo arenoso & 2 & Cerrado & Hatschbach, G. 31944 (NY) \\
\hline Calyptrocarya glomerulata (Brongn.) Urb. & Mata de galeria úmida & 11 & Floresta & Pott, V.J. 7005 (CGMS) \\
\hline Cladium jamaicense Crantz & & 18 & & Pott, V.J. 3805 (CGMS) \\
\hline Cyperus aggregatus (Willd.) Endl. & Campo limpo & 17 & Cerrado & Araújo, A.C. et al. 705 (ICN) \\
\hline C. andreanus Maury & Mata ciliar & 3 & Floresta & Pereira, M.C. 20 (CGMS) \\
\hline C. articulatus $\mathrm{L}$. & & 2 & Cerrado & Cunha, S.A. 367 (CGMS) \\
\hline C. chalaranthus J. Presl \& C. Presl & & 2 & Pantanal & Hatschbach, G. 58970 (MBM) \\
\hline C. compressus $\mathrm{L}$. & Campo & 8 & Pantanal & Sciamarelli, A. 1731 (CGMS) \\
\hline C. cornelii-ostenii Kük. & Afloramento rochoso & 13 & Cerrado & Bao, F. 101 (CGMS) \\
\hline C. corymbosus Rottb. & & 1 & Pantanal & Soares, V.C. 52. (CGMS) \\
\hline C. digitatus Roxb. & & 13 & Pantanal & Rodrigues, R.B. 07 (CGMS) \\
\hline C. distans L.f. & & 2 & Floresta & Penha, A.S. 467 (CGMS) \\
\hline C. eragrostis Lam. & Margem de mata (“coroa") & 4 & Floresta & Conceição, C.A. 1384 (CGMS) \\
\hline C. esculentus L. & Campo & 4 & Cerrado & Conceição, C.A. 2683 (CGMS) \\
\hline C. friburguensis Boeck. & & 3 & Cerrado & Kozera, C. 1786 (MBM) \\
\hline C. gardneri Nees & & 10 & Pantanal & Rodrigues, R.B. 70 (CGMS) \\
\hline C. giganteus Vahl & Local alagado & 107 & Floresta & Araújo, A.C. et al. 704 (ICN) \\
\hline C. haspan L. & Brejo próximo à represa & 32 & Floresta & Pott, V.J. 6981 (CGMS) \\
\hline C. imbricatus Retz. & & 7 & Pantanal & Bao, F.42 (CGMS) \\
\hline C. incomtus Kunth & & 10 & Cerrado & $\begin{array}{c}\text { Magalhães, L.C.S. } 135 \\
\text { (CGMS) }\end{array}$ \\
\hline C. iria L. & Área de cultivo & 10 & Ruderal & Araújo, A.C. et al. 708 (ICN) \\
\hline C. laxus Lam. & Cerrado & 14 & Cerrado & Hatschbach, G. 74936 (MBM) \\
\hline C. luzulae (L.) Retz. & Brejo & 23 & Ruderal & Araújo, A.C. et al. 712 (ICN) \\
\hline C. meyenianus Kunth & & 1 & Pantanal & Araújo, I.P.P. 4 (CGMS) \\
\hline C. ochraceus Vahl & Em solo palustre argiloso & 6 & Pantanal & Pott, V.J. 3826 (CGMS) \\
\hline C. odoratus L. & Campo de inundação & 34 & Pantanal & Araújo, A.C. et al. 716 (ICN) \\
\hline C. pohlii (Nees) Steud. & & 1 & Ruderal & $\begin{array}{c}\text { Damasceno Jr., G.A. } 4693 \\
\text { (CGMS) }\end{array}$ \\
\hline C. prolixus Kunth & Brejo & 14 & Ruderal & Hatschbach, G. 76996 (MBM) \\
\hline C. reflexus Vahl & & 4 & Cerrado & Pott, V.J. et al. 8566 (HMS) \\
\hline
\end{tabular}


Quadro 1. cont.

\begin{tabular}{|c|c|c|c|c|}
\hline Espécies & Habitat & Registros & Vegetação & Voucher \\
\hline C. rigens J. Presl \& C. Presl & & 2 & Cerrado & $\begin{array}{c}\text { Damasceno Jr., G.A. } 4466 \\
\text { (CGMS) }\end{array}$ \\
\hline C. rotundus $\mathrm{L}$. & Campo & 3 & Ruderal & Araújo, A.C. et al. 690 (ICN) \\
\hline Espécies & Habitat & Registros & Vegetação & Voucher \\
\hline C. schomburgkianus Nees & Lageados úmidos & 2 & Cerrado & Hatschbach, G. 23755 (NY) \\
\hline C. squarrosus $\mathrm{L}$. & & 1 & Pantanal & Pott, A. 7906 (ICN) \\
\hline C. surinamensis Rottb. & & 40 & Pantanal & Hatschbach, G. 77086 (MBM) \\
\hline C. uncinulatus Schrader \& Nees & & 3 & Pantanal & Hatschbach, G. 60814 (MBM) \\
\hline C. unicolor Boeck. & & 2 & Pantanal & Carvalho, F.S. 164 (CGMS) \\
\hline C. virens Michx. & Área de inundação & 7 & Pantanal & Duarte, G.S.V. 110 (CGMS) \\
\hline Eleocharis acutangula (Roxb.) Schult. & Brejo & 36 & & Pott, V.J. 7022 (CGMS) \\
\hline E. bicolor Chapm. & & 1 & & Pott, V.J. 7328 (CGMS) \\
\hline E. capillacea Kunth & & 7 & & Pott, V.J. 6915 (CGMS) \\
\hline E. elegans (Kunth) Roem. \& Schult. & & 51 & & Pott, V.J. 6767 (CGMS) \\
\hline E. filiculmis Kunth & & 56 & & Guglieri, A. 1596 (CGMS) \\
\hline E. flaveçãoscens (Poir.) Urb. & & 2 & & Hatschbach, G. 74110 (MBM) \\
\hline E. geniculata (L.) Roem. \& Schult. & & 31 & & Pott, V.J. 6815 (CGMS) \\
\hline E. hatscbachii R. Trevis. & & & & $\begin{array}{l}\text { Hatschbach, G. } 73128 \\
\text { (holótipo: MBM) }\end{array}$ \\
\hline E. interstincta (Vahl) Roem. \& Schult. & Orla do brejo & 41 & & $\begin{array}{l}\text { Damasceno Jr., G.A. } 1569 \\
\text { (CGMS) }\end{array}$ \\
\hline E. jelskiana Boeck. & & & & Salis, S.M. et al. 454 (LP) \\
\hline E. liesneri S.González \& Reznicek & & 1 & & Pott, A.6908 (CGMS) \\
\hline E. maculosa (Vahl) Roem. \& Schult. & & 1 & & Rego, A. 775 (CGMS) \\
\hline E. minima Kunth & & 44 & & Pott, V.J. 6814 (CGMS) \\
\hline E. montana (Kunth) Roem. \& Schult. & & 15 & & Pott, V.J. 7320 (CGMS) \\
\hline E. mutata (L.) Roem. \& Schult. & & 7 & & Pott, V.J. 7248 (CGMS) \\
\hline E. nudipes (Kunth) Palla & & 20 & & Sciamarelli, A. 923 (CGMS) \\
\hline E. obtusetrigona (Lindl. \& Nees) Steud. & & & & Pott, V.J. 6908 (CGMS) \\
\hline E. plicarhachis (Griseb.) Svenson & & 12 & & Pott, V.J. 7249 (CGMS) \\
\hline E. quinquangularis Boeck. & & 2 & & $\begin{array}{c}\text { Hatschbach, G. \& Callejas, R. } \\
47241 \text { (MBM) }\end{array}$ \\
\hline E. radicans (Poir.) Kunth & & 1 & & Hatschbach, G. 76498 (MBM) \\
\hline E. rugosa D.A.Simpson & & 3 & & $\begin{array}{l}\text { Vanzela, A.L.L. s.n. } \\
\text { (UFP12117) }\end{array}$ \\
\hline E. sellowiana Kunth & & 18 & & Araújo, A.C. et al. 737 (ICN) \\
\hline E. squamigera Svenson & & 1 & & Cordeiro, I. 1221 (SP) \\
\hline E. urceolatoides R. Trevis. \& Boldrini & & 1 & & Etchichury, L.M. 19 (SI) \\
\hline E. vivipara Link & & 1 & & Pereira, E. 512 (UB) \\
\hline Fimbristylis autumnalis (L.) Roem. \& Schult. & Beira de rio & 17 & Pantanal & Guglieri, A. 1586 (CGMS) \\
\hline F. complanata (Retz.) Link & & 11 & Pantanal & Rego, A. 612 (CGMS) \\
\hline F. dichotoma (L.) Vahl & Campo limpo & 42 & Pantanal & Araújo, A.C. et al. 688 (ICN) \\
\hline F. miliacea (L.) Vahl & & 1 & Pantanal & Hatschbach, G. 73140 (CGMS) \\
\hline F. squarrosa Vahl & Beira de rio & 2 & Pantanal & Pott, V.J. 3704 (CGMS) \\
\hline Fuirena incompleta Nees & & 5 & Pantanal & Pott, V.J. 7032 (CGMS) \\
\hline F. robusta Kunth & Ambiente alagado & 1 & Pantanal & $\begin{array}{c}\text { Kawakita, K. et al. } 251 \\
\text { (HUEM) }\end{array}$ \\
\hline F. umbellata Rottb. & & 22 & Pantanal & $\begin{array}{c}\text { Catharino, E..L.M. } 321782 \\
\text { (MBM) }\end{array}$ \\
\hline Isolepis hirtella Schrad. ex Schult. & & 18 & Cerrado & Conceição, C.A. 1961 (CAC) \\
\hline Kyllinga brevifolia Rottb. & & 6 & Ruderal & Guglieri, A. 1599 (CGMS) \\
\hline K. odorata Vahl & Campo & 12 & Pantanal & Conceição, C.A. 2431 (CGMS) \\
\hline K. pumila Michx. & & 2 & Ruderal & Guglieri, A. 1163 (CGMS) \\
\hline K. vaginata Lam. & Margens arenosas de rio & 6 & Ruderal & Araújo, A.C. et al. 694 (ICN) \\
\hline Lagenocarpus rigidus (Kunth) Nees & & & & possível ocorrência \\
\hline Lipocarpha humboldtiana Nees & & 9 & Pantanal & Carvalho, F.S. 102 (CGMS) \\
\hline \multicolumn{2}{|c|}{ L. micrantha (Vahl) G.C. Tucker (=Scirpus micranthus Vahl) } & 2 & Pantanal & Cervi, A.C. 4250 (UPCB) \\
\hline
\end{tabular}


Quadro 1. cont.

\begin{tabular}{|c|c|c|c|c|}
\hline Espécies & Habitat & Registros & Vegetação & Voucher \\
\hline Oxycaryum cubense (Poepp. \& Kunth) Palla & Ambiente alagado & 63 & Pantanal & Carvalho, F.S. 173 (CGMS) \\
\hline Pycreus flavescens (L.) Rchb. & Afloramento rochoso & 1 & Cerrado & Silva, R.H.940. (CGMS) \\
\hline $\begin{array}{l}\text { P. lanceolatus (Poir.) C.B. Clarke (=Cyperus } \\
\text { lanceolatus Poir.) }\end{array}$ & Ambiente alagado & 11 & Pantanal & Araújo, A.C. et al. 732 (ICN) \\
\hline P. megapotamicus (A. Dietr.) Nees & Brejo & 4 & Ruderal & Tucker, G. C. 25123 (NY) \\
\hline $\begin{array}{l}\text { P. niger (Ruiz \& Pav.) Cufod. (= Cyperus niger Ruiz } \\
\text { \& Pav.) }\end{array}$ & Ambiente alagado & 2 & Pantanal & Pott, V.J. 6904 (CGMS) \\
\hline P. tener C.B. Clarke & & 1 & Pantanal & $\begin{array}{l}\text { Damasceno Jr, G.A. } 4301 \\
\text { (CGMS) }\end{array}$ \\
\hline P. unioloides (R. Br.) Urb.(= Cyperus unioloides $\mathrm{R}$. Br.) & Brejo & 6 & Pantanal & Pott, V.J. 7340 (CGMS) \\
\hline Rhynchospora albiceps Kunth & Campo & 4 & Cerrado & $\begin{array}{l}\text { Araújo, A. C. et al. } 711 \text { (ICN, } \\
\text { SPF) }\end{array}$ \\
\hline R. albobracteata A. C. Araujo & Campo & 4 & Cerrado & provável ocorrência \\
\hline R. armerioides J.Pres1 \& C.Presl & Campo & 1 & Cerrado & provável ocorrência \\
\hline R. barbata (Vahl) Kunth & Borda de floresta & 3 & Floresta & Irwin, H.S. 16877 (MO) \\
\hline R. barrosiana Guagl. & & 1 & Cerrado & Pott, A. 6642 et al. (ICN) \\
\hline Espécies & Habitat & Registros & Vegetação & Voucher \\
\hline R. bracteovillosa A.C. Araujo \& W. W. Thomas & & & & provável ocorrência \\
\hline R. brevirostris Griseb. & & 1 & Pantanal & Schessl, M. 291 (CPAP) \\
\hline R. cephalotes (L.) Vahl & Cerrado & 4 & Cerrado & $\begin{array}{c}\text { Hatschbach, G. } 32408 \text { (MBM, } \\
\text { NY) }\end{array}$ \\
\hline R. ciliolata Boeck. & & & & provável ocorrência \\
\hline R. confusa Ballard & & & & provável ocorrência \\
\hline R. consanguinea (Kunth) Boeck. & Campo & 1 & Cerrado & Penha, A.S. 48 (CGMS) \\
\hline R. corymbosa $($ L.) Britton & Brejo & 44 & Pantanal & Pott, V.J. 3663 (F) \\
\hline R. elatior Kunth & Campo & 1 & Cerrado & Pedersen, T.M. 12233 (NY) \\
\hline R. emaciata (Nees) Boeck. & & 20 & Cerrado & $\begin{array}{c}\text { Hatschbach, G. } 58705 \text { (BHCB, } \\
\text { MBM) }\end{array}$ \\
\hline R. eurycarpa A.C. Araújo \& Longhi-Wagner & Campo cerrado aberto & 2 & Cerrado & $\begin{array}{c}\text { Hatschbach, G. \& Guimarães, } \\
\text { O. } 24635 \text { (MBM) }\end{array}$ \\
\hline R. exaltata Kunth & & 17 & Cerrado & $\begin{array}{l}\text { Hatschbach, G. \& Guimarães, } \\
\text { O. } 24535 \text { (MBM, NY) }\end{array}$ \\
\hline R. exilis Boeck. & & & & possível ocorrência \\
\hline R. eximia (Nees) Boeck. & Aquática & 4 & Pantanal & Pott, A. 3203 (CPAP) \\
\hline R. globosa (Kunth) Roem. \& Schult. & Brejo & 21 & Cerrado & Dambros, L. A. 202 (HRB) \\
\hline R. hassleri C.B. Clarke & Campo & 4 & Cerrado & $\begin{array}{c}\text { Hatschbach, G. } 76983 \text { (INPA, } \\
\text { MBM) }\end{array}$ \\
\hline R. hirta (Nees) Boeck. & Campo & 6 & Cerrado & $\begin{array}{c}\text { Hatschbach, G. } 32346 \text { (MBM, } \\
\text { NY) }\end{array}$ \\
\hline R. holoschoenoides (Rich.) Herter & Do pantanal & 4 & Pantanal & $\begin{array}{c}\text { Hatschbach, G. } 29575 \text { (MBM, } \\
\text { NY) }\end{array}$ \\
\hline R. junciformis (Kunth) Boeck. & Campo & 1 & Cerrado & $\begin{array}{l}\text { Araujo, A. C. et al. } 739 \text { (ICN, } \\
\text { SPF) }\end{array}$ \\
\hline R. loefgreni Boeck. & campo & 2 & Cerrado & Guimaraes, J. G.1333 (RB) \\
\hline R. longa (Lindm.) Pfeiff. & margem de rio & & & possível ocorrência \\
\hline R. melanocarpa A.C. Araujo \& W.W. Thomas & & & & possível ocorrência \\
\hline R. nervosa (Vahl) Boeck. & Campo & 12 & Cerrado & $\begin{array}{l}\text { Menstedt, E.F. s.n. (RB } \\
\text { 231949) }\end{array}$ \\
\hline R. patuligluma C.B. Clarke & & & & provável ocorrência \\
\hline R. pilosa Boeck. & & & & provável ocorrência \\
\hline R. polyphylla (Vahl) Vahl & & & & possível ocorrência \\
\hline R. pubera (Vahl) Boeck. & Campo & 1 & Cerrado & $\begin{array}{l}\text { Catharino, E.L.M. et al. } 1790 \\
\text { (ICN) }\end{array}$ \\
\hline \multicolumn{4}{|l|}{ R. radicans ssp. microcephala (Botero ex Sprengel) Thomas } & provável ocorrência \\
\hline \multicolumn{4}{|l|}{ R. reptans (L.C. Rich.) Boeck. } & provável ocorrência \\
\hline R. riparia (Nees) Boeck. & Cerrado, margem de rio & 2 & Cerrado & Ratter, J.A. 3310 (NY) \\
\hline R. robusta (Kunth) Boeck. & & 4 & Pantanal & Pott, J.V. 4078 (CPAP) \\
\hline
\end{tabular}


Quadro 1. cont.

\begin{tabular}{|c|c|c|c|c|}
\hline Espécies & Habitat & Registros & Vegetação & Voucher \\
\hline R. rugosa (Vahl) Gale & Campo & 7 & Cerrado & Pott, J.V. 3685 (CPAP) \\
\hline R. rupestris A. C. Araujo \& W. W. Thomas & Campo & 1 & Cerrado & $\begin{array}{l}\text { Hatschbach, G. } 58709 \\
\text { (MBM, MO) }\end{array}$ \\
\hline R. scutellata Griseb. & Da beira de lagoa & 8 & Pantanal & $\begin{array}{l}\text { Hatschbach, G. } 29582 \\
\text { (MBM, NY) }\end{array}$ \\
\hline R. setigera Griseb. & campo & 3 & Cerrado & $\begin{array}{l}\text { Pedersen, T.M. } 14766 \text { (CTES, } \\
\text { MO) }\end{array}$ \\
\hline R. speciosa (Kunth) Boeck. & Afloramentos rochosos. & 1 & Cerrado & $\begin{array}{l}\text { Catharino, E.L.M. et al. } 1772 \\
\text { (NY, SP) }\end{array}$ \\
\hline R. spruceana C.B. Clarke & & 1 & Cerrado & Pott, V.J. et al. 6302 (ICN) \\
\hline R. tenerrima Nees ex Spreng. & Campo umido. & 1 & Cerrado & Pott, A. 3407 (CPAP) \\
\hline R. tenuis Link & Brejo & 21 & Cerrado & $\begin{array}{l}\text { Catharino, E..L.M. } 321772 \\
\text { (NY) }\end{array}$ \\
\hline R. terminalis (Nees) Steud. & & & & provável ocorrência \\
\hline R. triflora Vahl & Brejo & 3 & Cerrado & $\begin{array}{l}\text { Hatschbach, G. } 23879 \\
\text { (MBM, NY) }\end{array}$ \\
\hline R. trispicata (Nees) Schrad. ex Steud. & Margem de lago & 20 & Cerrado & Schaller, G. 138 (NY) \\
\hline R. velutina (Kunth) Boeck. & Brejo & 24 & Cerrado & Catharino, E.L.M. 1777 (UFP) \\
\hline R. warmingii Boeck. & & & & provável ocorrência \\
\hline Schoenoplectiella supina (L.) Palla & & 3 & Pantanal & Pott, A. 3673 (CPAP) \\
\hline Scleria bracteata Cav. & Cerrado, margens rio & 4 & Cerrado & $\begin{array}{c}\text { Hatschbach, G. } 24532 \text { (INPA, } \\
\text { MBM) }\end{array}$ \\
\hline S. comosa (Nees) Steud. & & 1 & Pantanal & Hatschbach, G. 24260 (NY) \\
\hline S. cyperina Willd. ex Kunth & & 1 & Pantanal & Resene, U.M. 1406 (CGMS) \\
\hline S. distans Poir (=S. nutans Willd. ex Kunth) & Campo úmido & 1 & Cerrado & $\begin{array}{c}\text { Hatschbach, G. } 24989 \text { (MBM, } \\
\text { NY) }\end{array}$ \\
\hline S. gaertneri Raddi & Cerradao & 8 & Cerrado & Bommer, D. 41 (NY) \\
\hline S. hirtella Sw. & Brejo & & Cerrado & Pott, V.J. 7670 (CPAP) \\
\hline S. interrupta Rich. & & & & provável ocorrência \\
\hline S. latifolia Sw. & Margens rio, local brejoso & 3 & Floresta & Hatschbach, G. 35945 (NY) \\
\hline S. lacustris C. Wright & & 2 & Pantanal & Rodrigues, R.B. 43 (CGMS) \\
\hline S. longigluma Kük. & & 1 & Cerrado & Nuenstedt. E.F. s.n (RB) \\
\hline S. leptostachya Kunth & Campo cerrado & 5 & Cerrado & Pott, V.J. 10255 (CPAP) \\
\hline S. macrophylla J. Presl \& C. Presl & Borda de mata & 4 & Pantanal & Pott, V.J. 5349 (CPAP) \\
\hline S. martii (Nees) Steud. & Solo arenoso & 2 & Floresta & Hatschbach, G. 33191 (NY) \\
\hline S. microcarpa Nees ex Kunth & Cerrado & 11 & Cerrado & Pott, V.J. 6588 (CGSM) \\
\hline S. mitis P. J. Bergius & Mata galeria & 11 & Florestal & Araújo, A.C. et al 696. (ICN) \\
\hline S. panicoides Kunth & Mata de galeria & 1 & Florestal & $\begin{array}{l}\text { Hatschbach, G. } 25203 \\
\text { (MBM, NY) }\end{array}$ \\
\hline S. plusiophylla Steud. & & 2 & Pantanal & Silva, R.H. 841 (CGMS) \\
\hline S. ramosa C.B. Clarke & & 1 & Pantanal & $\begin{array}{l}\text { Hatschbach, G. } 74110 \\
\text { (MBM, MO) }\end{array}$ \\
\hline S. scabra Willd. & & & & provável ocorrência \\
\hline S. secans (L.) Urb. & Do cerrado & 2 & Cerrado & Hatschbach, G. 31900 (NY) \\
\hline $\begin{array}{l}\text { S. testacea Nees ex Kunth (= S. phylloptera C. Wright } \\
\text { ex Griseb.) }\end{array}$ & Campo de várzea & 1 & Pantanal & Catharino, E.L.M. 1796 (NY) \\
\hline S. uleana Boeck. ex C.B. Clarke & & 1 & Pantanal & $\begin{array}{l}\text { Kawakita, K. et al. } 1534 \\
\text { (HUEM) }\end{array}$ \\
\hline S. variegata (Nees) Steud. & Mata ciliar & 2 & Pantanal & Pott, V.J. 6392 (CGSM) \\
\hline S. verticillata Muhl. ex Willd. (= S. cuyabensis Pilg.) & Campo & & Cerrado & provável ocorrência \\
\hline
\end{tabular}

Alves et al. (2009) afirmam que estes gêneros apresentam maior diversidade em ambientes florestais e disjunto entre a floresta Amazônica e Atlântica. Contudo, algumas espécies apresentam ampla distribuição e foram registradas tanto no domínio Amazônico quanto no domínio Atlântico, a saber: Hypolytrum pulchrum (Rudge) H. Pfeiff., H. schraderianum
Nees, Pleurostachys beyrichii (Nees) Steud. e P. puberula Boeck. Seria de se esperar a ocorrência destes taxa em floresta de galeria que cortam o Mato Grosso do Sul. No cerrado, porém, o gênero Hypolytrum não é tão diverso; as seguintes espécies foram citadas para o Centro-Oeste: $H$. longifolium (Rich.) Nees, H. rigens Nees e H. stemonifolium 


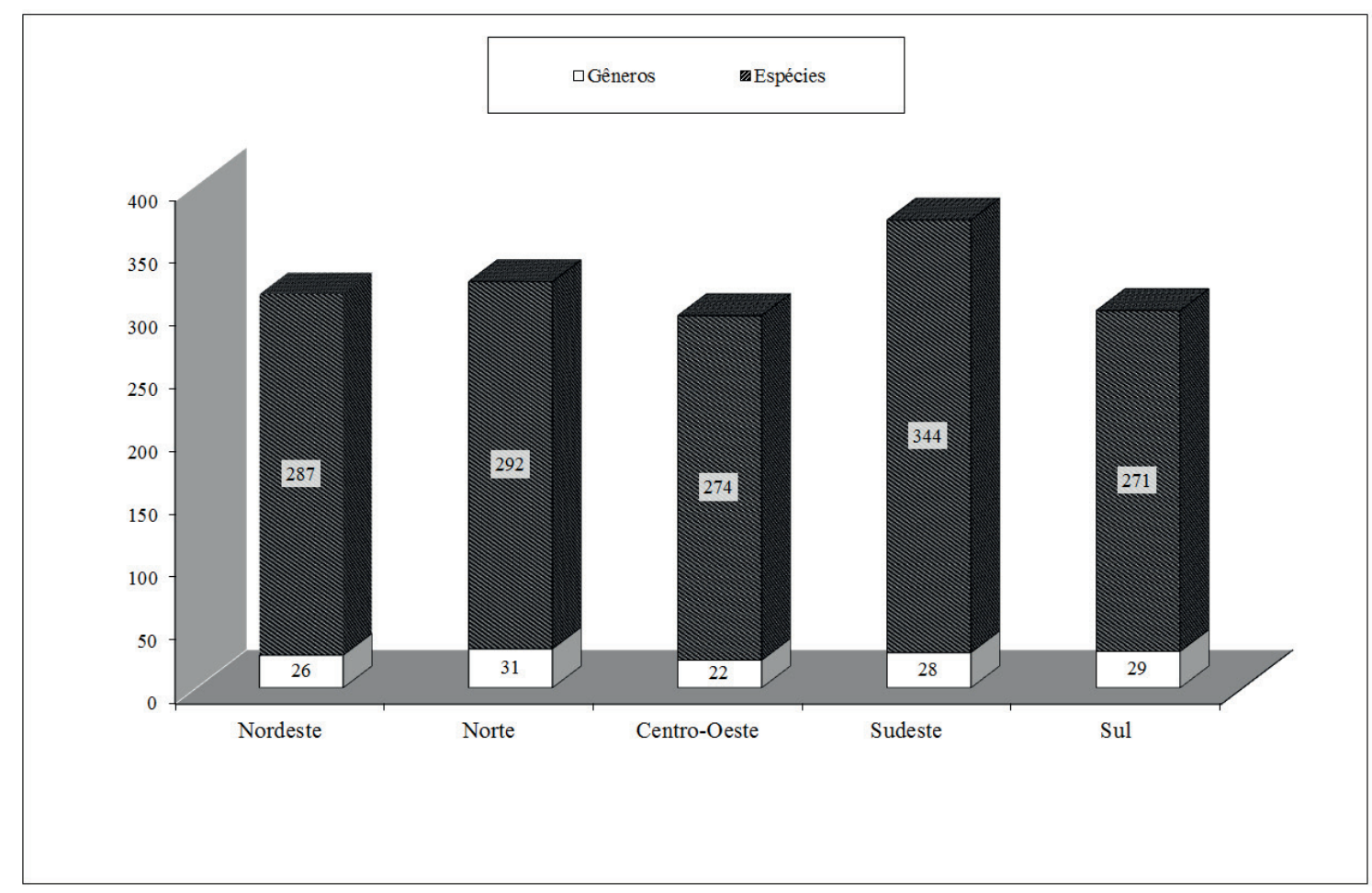

Fig. 1. Riqueza de espécies por gênero de Cyperaceae no estado do Mato Grosso do Sul.

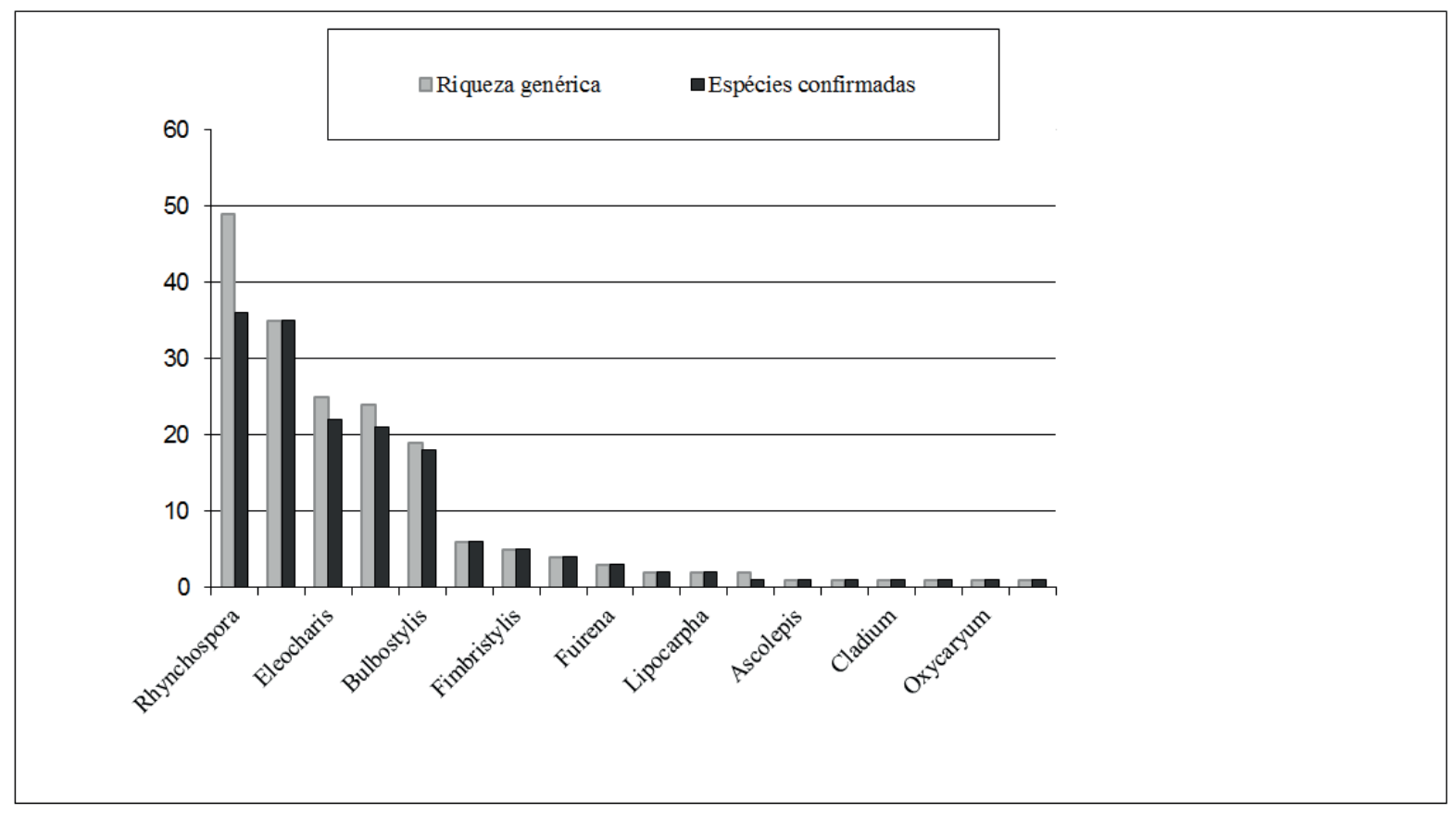

Fig. 2. Riqueza de gêneros e espécies de Cyperaceae por regiao brasileira (Alves et al. 2013).

T. Koyama; porém nenhuma delas foi referida ou registrada neste estado até o momento. Pleurostachys puberula é amplamente distribuida na América do Sul, registrada de norte a sul no continente desde os Andes até a costa Atlântica, está referida para o Centro-Oeste, porém sem registro para o Mato Grosso do Sul.
Por outro lado, estranha-se a referência de Diplacrum capitatum (Wild.) Boeck. para o Mato Grosso do Sul (Alves et al. 2014) visto que a espécie é de domínio amazônico, consequentemente amplamente distribuída na região Norte do Brasil e presente no estado do Mato Grosso (Centro-oeste) e com possível 


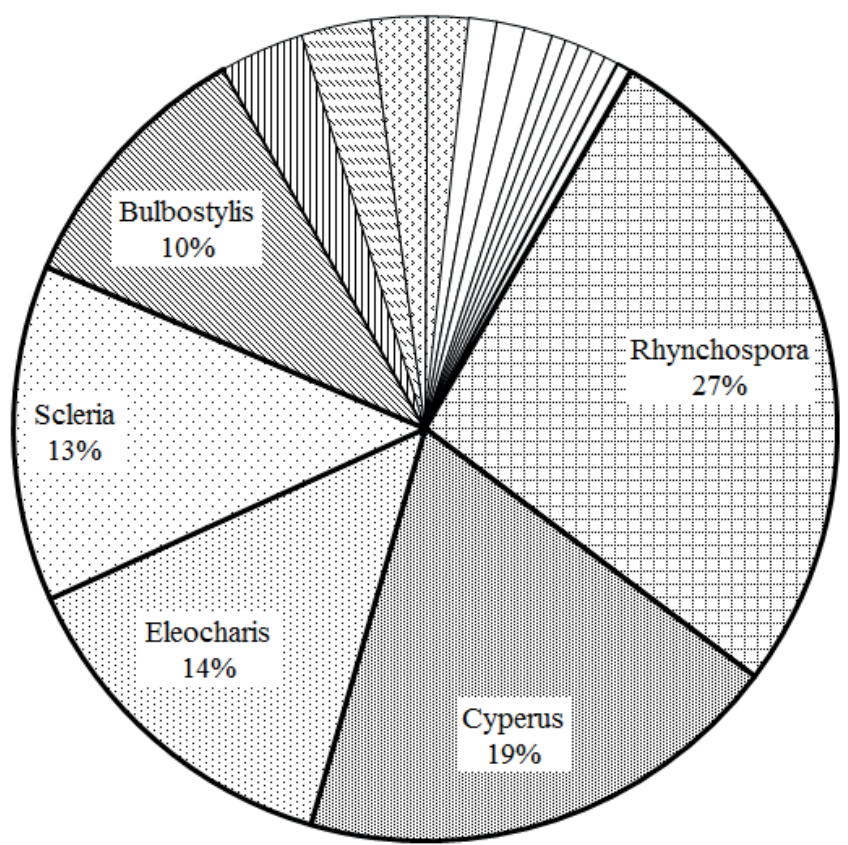

Fig. 3. Porcentagem de espécies encontrada nos gêneros mais diversos da flora do Mato Grosso do Sul.

ocorrência também no estado do Maranhão (Nordeste).

É importante ressaltar que os comentários relacionados à diversidade vegetacional ainda são preliminares, resultado das informações fornecidas pelos herbários, onde em alguns casos estão incompletas e são insuficientes para documentar precisamente a diversidade florística e vegetacional no estado.

Com base no presente resultado, considera-se relevante uma reavaliação da listagem da flora do estado do Mato Grosso. Acredita-se que o número de espécies e gêneros ora listados para aquele estado aumentaria, visto que esse inclui vegetação de floresta Amazônica, ausente no Mato Grosso do Sul, para a qual a ocorrência de diversos outros gêneros de Cyperaceae é restrita (Alves et al. 2009).

Cabe lembrar que algumas espécies aqui citadas aparecem sinonimizadas em Govaerts \& Simpson (2007), como por exemplo Albidgaardia ovata (Burm. f.) Kral e Bulbostylis hirtella (Schrad. ex Schult.) Nees ex Urb. Estudos recentes, no entanto, indicam que ambos os taxa merecem o status de "espécie aceita" (Prata 2004).

Acredita-se que um maior esforço de coleta no estado bem como a identificação completa das coleções existentes aumentaria a riqueza de espécies confirmadas para a flora do Mato Grosso do Sul, particularmente as espécies esperadas porém até o memento não confirmadas durante a execução deste estudo ou de outros estudos similares.

\section{Taxonomia e informações nomenclaturais}

Scleria verticillata Muhl ex Willd. Sp. Pl. 4: 317. Tipo. Estados Unidos: Virgina, North Carolina, Willdenow 17326 (holótipo: B; isótipo W!, foto do holótipo US!). = Scleria cuyabensis Pilg., Bot. Jabrb. 30: 144. 1901.
Tipo. Brasil: Mato Grosso, "haufig zerstreut auf trocknem, steinigen Boden bei Cuyaba", Mar.1899, Mayer 360 (holótipo: B; isótipo: NY!, foto do holótipo F- F0BN011202). Sin. nov.

Aqui propõe-se Scleria cuyabensis Pilg. como sinônimo de Scleria verticillata Muhl ex Willd. O estudo cuidadoso dos protólogos e a comparação dentre coleções de materialtipo destes nomes prova que ambos referem-se ao mesmo táxon. Core (1936) já havia referido que S. cuyabensis e $S$. verticillata eram próximas, diferenciando-se somente pelo tamanho das espiguetas, maior na primeira. Mais recentemente, Camelbeke \& Goetghebeuer (2002), Camelbeke et. al. (2003) ao investigar as floras de Colômbia e Bolívia descreveu $S$. verticillata com variabilidade morfológica mais ampla, amplitude esta que inclui os caracteres taxonômicos usados para distinguir $S$. cuyabensis de $S$. verticillata, tornando-os irrelevante.

\section{Principais Acervos}

Até o momento os principais acervos da flora Sulmatogrossense estão concentrados nos herbários: CGMS, HMS, MBM, NY e SPF. Contudo, é relevante considerar as coleções dos herbários BHCB, E, LPB, MO, R, RB, UB, UFMT, UFP onde a flora Sul-matogrossense está bem representada principalmente em anos mais recentes.

Em termos de conhecimento da flora ciperológica nacional os herbários brasileiros mais representativos são: ICN, INPA, R, RB, K, SPF e UEC, sendo o INPA o que melhor representa a vegetação amazônica. No exterior as coleções mais importantes e/ou numerosas, incluindo seu valor histórico, estão concentradas em B, BR, C, K, LE, M, MA, NY, P, S e U. 


\section{Perspectivas de Pesquisa para o Grupo nos próximos 10 anos}

Obter o conhecimento específico da flora ciperológica no Brasil deveria ser a prioridade. Esta meta seria mais facilmente obtida se estudos florísticos regionais e estudos taxonômicos no nível genérico fossem promovidos de forma mais efetiva. É de inestimável valor conhecer: a) o número de espécies e respectiva distribuição; b) a dinâmica e estado de conservação das populações; c) os endemismos presentes; d) a qualidade e resiliência da vegetação onde estas populações ocorrem e, finalmente, entender a interação entre tais populações de forma a contribuir com o entendimento da formação história dos biomas e sua importância na variação climática tão discutida atualmente. Infelizmente, as investigações florísticas que contribuiríam para um completo entendimento de riqueza, diversidade e conservação vegetal não encontram fácil receptividade para publicação.

Como mencionado acima, são os resultados de mestrado e dourado que mais contribuem para o conhecimento florístico. Porém, para manterem-se ativos os cursos de pós-graduação precisam seguir as regras da Coordenação de Aperfeiçoamento de Pessoal de Nível Superior, entre as quais a produção científica é uma das mais relevantes. A produção científica necessariamente precisa estar alinhada aos veículos científicos de comunicação com alto fator de impacto (Qualis A e B). Infelizmente são poucos as revistas científicas no nível exigido que se dispõe a publicar resultados florísticos. Em sua maioria, as revistas científicas que dedicam-se a publicar resultados florísticos possuem fator de impacto inaceitável para a CAPES. A pressão enfrentada pelos profissionais causa ansiedade em relação ao futuro dos cursos de pós-graduação direcionando-os para as áreas mais atrativas no momento da publicação.

Ressalta-se também que revistas que aceitam a publicação de trabalhos extensos são as mais buscadas pelos taxonomistas, consequentemente a espera na publicação é maior, e também os riscos de, em se esperar anos pela publicação, por um lado os resultados já estarão desatualizados; por outro, no todo ou em parte, dados originais muitas vezes já são de conhecimento público via eletrônica sem a concordância do autor principal e sem necessariamente esclarecer a fonte de onde os mesmos foram obtidos. Toda esta situação certamente impõe nos editores e revisores uma quantidade extra de trabalho, os quais deveriam estar dedicando tempo a suas próprias investigações; paralelamente esta mesma situação requer paciência e confiança dos autores na comissão estabelecida pela revista para onde o manuscrito foi submetido, no sentido de agilizar a publicação, antes que dados originais se tornem obsoletos.

A dificuldade na publicação impressa de estudos florísticos, o longo tempo que escorre entre o momento da submissão e o da efetiva publicação (o qual este estudo é um exemplo), aliados ao evento de "listas florísticas publicadas eletronicamente"- as quais, sendo mais rápidas, muitas vezes absorvem os dados de outros autores que estão presos ao sistema moroso da publicação impressa- desistimulam o interesse de taxonomistas em promover estudos florísticos. Soma-se ainda a constante publicação de novos taxa nos mais diferentes níveis taxonômicos com base, muitas vezes exclusivamente, em estudos moleculares, provocando um aumento desnecessário de lista de sinônimos e retardando o avanço dos estudos botânicos fundamentais no efetivo progresso e desenvolvimento da ciência.

No que diz respeito a ciperologia brasileira prevalece como prioridade o estudo florístico, riqueza e diversidade de espécies, particularmente para as regiões Norte e Centrooeste; bem como entender preferência vegetacional das espécies, adaptação ao meio, estratégia de dispersão e de ocupação ambiental, enfocando as possíveis respostas a mudanças climáticas vigentes. Além disso, considerase importante busca contínua de entender as relações filogenénicas entre grupos aliados dentro de Cyperaceae, bem como desta família em relação aos seus aliados dentro de Poales.

\section{AGRADECIMENTOS}

À Dra. Maria Ana Farinaccio pelo constante suporte durante a elaboração deste artigo. A todos os curadores e/ ou pesquisadores ligados aos herbários aqui citados por facilitar o acesso às informações. Ao Museu de História Natural de Londres, particularmente ao Dr. Neil Brummitt, pela gentileza no suporte técnico e científico durante o desenvolvimento deste estudo, bem como à equipe de curadores. Aos demais colegas ciperológos que gentilmente atenderam nossas solicitações. À Synthesis of Systematic Resources, pelo apoio financeiro durante a visita ao herbário de Botanischer Garten und Botanisches Museum BerlinDahlem quando estudou-se a coleção da flora ciperológica da região neotropical.

\section{REFERÊNCIAS}

Alves, M.V. 2003. Hypolytrum Rich. (Cyperaceae) nos Neotrópicos. Tese 163p. Universidade de São Paulo. São Paulo.

Alves, M.V., Araújo, A.C., Prata, A.P., Vitta, F.A., Hefler, S.M., Trevisan, R., Gil, A.B., Martins, S. \& Thomas, W.W. 2009. Diversity of Cyperaceae in Brazil. Rodriguésia 60: 771-782.

Alves, M., Araújo, A.C., Hefler, S.M., Trevisan, R., Silveira, G.H. \& Luz, C.L. 2013. Cyperaceae. In Lista de Espécies da Flora do Brasil. Jardim Botânico do Rio de Janeiro. Disponível em: http://floradobrasil.jbrj. gov.br/2012/FB000100. Acessado em 25.10.2013.

Alves, M., Hefler, S.M., Trevisan, R., Silveira, G.H. \& Luz, C.L. 2014. Cyperaceae. In Lista de Espécies da Flora do Brasil. Jardim Botânico do Rio de Janeiro. Disponível em: http://floradobrasil.jbrj.gov. br/2012/FB000100. Acessado em 18.07.2014.

.2015. Cyperaceae. In Lista de Espécies da Flora do Brasil. Jardim Botânico do Rio de Janeiro. Disponível em: http://floradobrasil.jbrj. gov.br/2012/FB000100. Acessado em 29.08.2015.

Afonso, R., Zannin, A., Brummitt, N.A. \& Araújo, A.C. 2015. Diversity of Scleria (Cyperaceae) in Santa Catarina, Brazil. Rodriguesia 66: 353-367.

Araújo, A.C., Prata, A.P., Oliveira, A.R., Alves, M., Trevisan, R. \& Hefler, S.M. 2009. Cyperaceae. In Flora do Distrito Federal, Brasil (M.F. Batista \& T.B. Cavalcanti, eds.) Embrapa, Distrito Federal, v.7, p. 43-187. 
Angiosperm Phylogeny Group 2009. An update of the Angiosperm Phylogeny Group classification for the orders and families of flowering plants: APG III. Botanical Journal of the Linnean Society 161(2): $105-121$

Bruce, A.F., Naczi, R.C. \& Starr, J.R. 2008. Carex sect. Phyllostachyae: the value of a multidisciplinary approach in conducting systematics studies in sedges. In Sedges: Uses, diversity and systematics of the Cyperaceae (F.C. Naczi \& B.A. Ford, eds.). Missouri Botanical Garden Press, St. Louis. p. 227-242.

Brummitt, R.K. \& Powell, C.E. 1992. Authors of plant names. Royal Botanic Gardens. Kew. 732p.

Camelbeke, K. \& Goetghebeuer, P. 2002. The genus Scleria (Cyperaceae) in Colombia - An updated checklist. Caldasia 24: 259-268.

Camelbeke, K., Spruyt, K. \& Goetghebeur, P. 2003. The genus Scleria (Cyperaceae) in Bolívia. Revista de la Sociedad Boliviana de Botánica 4: $139-170$.

Core, E.L. 1936. The American species of Scleria. Brittonia 2: 1-105.

Dubs, B. 1998. Podromus florae Matogrossensis - Checklist of Angiosperms 3. Betrona-Verlag, Küsnacht, Switzerland. 444p.

Goetghebeur, P. 1998. Cyperaceae. In The families and genera of vascular plants (K. Kubitzki, ed.). Koeltz Botanical Books, Oberreifenberg 4: $141-190$.

Govaerts, R., \& Simpson, D.A. 2007. World Checklist of Cyperaceae. Facilitated by the Royal Botanic Gardens, Kew. Disponível em: http://apps.kew.org/wcsp/. Acessado em 16.11.2012.
Heywood, V.W., Brummitt, R.K., Culham, A. \& Seberg, O. 2007. Flowering Plant Families of the World. Royal Botanic Gardens, Kew. $424 \mathrm{p}$.

Lorenzi, H. 2006. Plantas daninhas do Brasil. Instituto Plantarum. São Paulo. 672p.

Pott, V. \& Pott, A. 2000. Plantas Aquáticas do Pantanal. Empresa Brasileira de Pesquisa Agropecuaria, Comunicação para traransferência de tecnologia. EMBRAPA, Campo Grande. 404p.

Prata, A. 2004. Bulbostylis Kunth (Cyperaceae) no Brasil. Tese 197p., Universidade de São Paulo, São Paulo.

Rodrigues, A.C. \& Estelita, M.E.M. 2002.Primary and secondary development of Cyperus giganteus Vahl rhizome (Cyperaceae). Revista Brasileira de Botânica 25: 251-258.

Simpson, D.A. 1998. Cyperaceae. In Podromus florae Matogrossensis (B. Dubs, ed.), Betrona-Verlag, South Lyon 3: 81-86.

2008. Frosted curls to tiger nuts: ethnobotany of Cyperaceae. In Sedges: Uses, diversity and systematics of the Cyperaceae (F.C. Naczi \& B.A. Ford, eds.) Missouri Botanical Garden Press, St. Louis. p. 1-14.

Thiers, B. 2012[continuously updated]. Index Herbariorum: A global directory of public herbaria and associated staff. New York Botanical Garden's Virtual Herbarium. Disponível em: http://sweetgum.nybg. org/ih/. Acessado em 16.11.2012.

Thomas, W.W. 2004. Cyperaceae. In Flowering Plants of the Neotropics (N. Smith, S.A. Mori, A. Henderson, D.W. Stevenson, \& S.V. Heald, eds.). Princeton University Press, Princeton p. 434-436. 